\title{
Profiling of exosomal microRNAs expression in umbilical cord blood from normal and preeclampsia patients
}

\author{
Hai-Tao Pan ${ }^{1,2,3}$, Xiao-Liang Shi ${ }^{1,2}$, Min Fang 1,2, Xiang-Mei Sun ${ }^{2}$, Pan-Pan Chen ${ }^{2}$, Jin-Long Ding ${ }^{1,2}$, Gui-Yu Xia ${ }^{1,2}$, \\ Bin $\mathrm{Yu}^{1,2}$, Tao Zhang ${ }^{1,2^{*}}$ and Hong-Dan Zhu ${ }^{1,2^{*}}$
}

\begin{abstract}
Background: Epidemiological and experimental studies suggest that preeclampsia has a negative impact on maternity and offspring health. Previous studies report that dysregulation in utero-environment increases risk for elderly disease such as cardiovascular disease. However, the underlying mechanisms remain elusive. Specific microRNAs (miRNAs) are packaged in exosomes may regulate microvascular dysfunction in offspring of mothers with preeclampsia. The present study aimed to identify the differential expression profiles of microRNAs in the serum exosomes between patients with preeclampsia and normal pregnancies.
\end{abstract}

Methods: A comprehensive miRNA sequence-based approach was performed to compare exosomes carry miRNAs (Exo-miRNAs) expression levels in umbilical serum between normal and preeclampsia patients. Exosomes were isolated using the ExoQuick precipitation kit. Serum exosomes were then viewed under electron microscopy, and their characteristics determined by western blotting and nanoparticle-tracking analysis. Illumina platform was used to perform sequencing. Bioinformatics analysis was used to explore differentially expressed Exo-miRNAs in umbilical serum.

Results: Based on sequence similarity, 1733 known miRNAs were retrieved. Furthermore, 157 mature miRNAs in serum exosomes were significantly differential expressed between PE and those control groups $\left(P<0.05, \log _{2}|F C|>1\right)$. Out, of the 157 miRNAs, 96 were upregulated miRNAs whereas 61 miRNAs were downregulated. The 157 differentially expressed miRNAs targeted 51,424 differentially expressed genes. Functional analysis through KEGG pathway and Gene Ontology results uncovered that target genes of miRNAs with differential expression were significantly linked to several pathways and biological processes.

Conclusion: The findings of this study showed differential expression of umbilical serum Exo-miRNAs in normal compared with PE patients, implying that these Exo-miRNAs may associate with microvascular dysfunction in offspring of mothers with preeclampsia.

Keywords: Preeclampsia, Umbilical serum, Exosomal miRNAs, Offspring, Microvascular dysfunction, Gamete and embryo-fetal origins of adult diseases

*Correspondence: zt801025@usx.edu.cn; zhuhongdan@sxfby.com

1 Shaoxing Maternity and Child Health Care Hospital, Shaoxing 312000, China

Full list of author information is available at the end of the article

\section{Background}

Preeclampsia (PE) is a complication of pregnancy. PE is associated with initiation of hypertension after 20 weeks of gestation accompanied by proteinuria, and affects about $5 \%$ of pregnant mothers [1]. Previous studies report that women with PE are likely to develop cardiovascular 
diseases [2]. In addition, offspring of mothers with preeclampsia have higher risk of cardiovascular disease [3].

Exosomes are membrane-bound vesicles released from all cell types into body fluids including plasma, urine, saliva and malignant effusions [4]. The size of exosomes ranges between 30 and $150 \mathrm{~nm}$ in diameter. Studies on exosomes show that they are implicated in modulating intercellular communication [5]. Exosomes secrete substances such as proteins, lncRNA, mRNA and microRNAs that are involved in regulation cell function [6]. Epigenetic modifications including alterations in microRNA levels are implicated in development of cardiovascular disease [7]. MicroRNAs (miRNAs) are involved in the modulation of key cellular activities, e.g., proliferation, metabolism, immune activities, apoptosis, neurodevelopment and epigenetics [8]. Impaired maternal cardiovascular function (cerebrovascular and hidden cardiovascular diseases) and dysfunctional placenta may cause epigenetic changes due to pregnancy-related complications in umbilical cord blood. These changes may increase risk of cerebrovascular and cardiovascular diseases in the newborn [9]. The 'Gamete and Embryo-fetal Origins of Adult Diseases' hypothesis states that patterns of fetal growth and development are implicated in risks to chronic non-communicable diseases in adult life [10]. However, development of altered vascular function and endothelial dysfunction in offspring has not been fully explored.

Previous studies report that PE increases risk of cardiovascular disease in pregnant mothers [11].In addition, PE causes persistent vascular dysfunction in pulmonary and systemic circulation of the offspring [12]. However, studies have not carried out high throughput sequencing of microRNAs in umbilical cord blood exosomes in women with or without preeclampsia have not been attempted.
The current study sought to generate an exosomal microRNAs map of umbilical cord blood in preeclampsia.

\section{Materials \& methods \\ Study participants}

Umbilical serum from normal and mild preeclampsia patients were collected after cesarean deliveries at the Shaoxing Maternity and Child Health Care Hospital. The Ethics Committee of Shaoxing Maternity and Child Health Care Hospital approved the study (Ethical approval no. 2018022). All subjects enrolled in this study provided a written informed consent. Umbilical sera were collected from healthy individuals $(n=5)$ and PE patients $(n=5)$. Inclusion criteria for the study were: singleton pregnancy, full-term delivery, maternal age between 25 and 35 years, diastolic blood pressure $\geq 90 \mathrm{mmHg}$ and/or systolic blood pressure $\geq 140 \mathrm{mmHg}$, normal birth weight ranging between 2500 and $4000 \mathrm{~g}$, no known birth or other complications. Normal control subjects were matched with PE women for maternal age, gestational weeks, gravidity and parity. Detailed patient characteristics are further outlined in Table 1.

\section{Exosomes purification and characterization}

Samples were sieved using $0.22-\mu \mathrm{m}$-pore-size filters following a method describedpreviously [13]. After precipitation of exosomes with the ExoQuick Exosome Precipitation Solution (System Biosciences-SBI), they were analyzed using an electron microscope (FEI Quanta 250, Thermo Fisher Scientific, Eindhoven, the Netherlands), the Nano particle tracking analysis (NTA) (ZetaView PMX 110, Particle Metrix, Meerbusch, Germany), and Western blot analysis (for CD63) were used to characterize isolated exosomes.

Table 1 The clinical data of the patient and control groups

\begin{tabular}{|c|c|c|c|c|c|c|c|c|c|}
\hline & Sample name & $\begin{array}{l}\text { Maternal } \\
\text { age }(y)\end{array}$ & $\begin{array}{l}\text { Gestational } \\
\text { age (wk) }\end{array}$ & $\begin{array}{l}\text { Birth weight } \\
\text { (g) }\end{array}$ & Gender & $\begin{array}{l}\text { Maternal } \\
\text { systolic/ } \\
\text { diastolic blood } \\
\text { pressure (mm } \\
\mathrm{Hg})\end{array}$ & Proteinuria & $\begin{array}{l}\text { Maternal } \\
\text { fasting blood } \\
\text { glucose } \\
\text { (mmol/L) }\end{array}$ & Diagnosis \\
\hline \multirow[t]{5}{*}{ Control } & $\mathrm{C} 1$ & 27 & 38 & 3600 & $M$ & $119 / 73$ & - & 4.88 & Normal \\
\hline & $\mathrm{C} 2$ & 30 & 37 & 3500 & $M$ & $110 / 68$ & - & 4.93 & Normal \\
\hline & C3 & 29 & 39 & 3400 & $M$ & $123 / 75$ & - & 4.91 & Normal \\
\hline & C4 & 28 & 37 & 3650 & $\mathrm{~F}$ & $125 / 76$ & - & 5.03 & Normal \\
\hline & C5 & 28 & 40 & 3550 & $\mathrm{~F}$ & $112 / 73$ & - & 4.86 & Normal \\
\hline \multirow[t]{5}{*}{ Preeclampsia } & T1 & 28 & 38 & 3450 & $M$ & $152 / 86$ & ++ & 5.08 & Preeclampsia \\
\hline & $\mathrm{T} 2$ & 30 & 37 & 3300 & $M$ & $149 / 78$ & ++ & 5.03 & Preeclampsia \\
\hline & T3 & 31 & 37 & 3250 & $M$ & $150 / 75$ & ++ & 4.96 & Preeclampsia \\
\hline & $\mathrm{T} 4$ & 27 & 37 & 3400 & $\mathrm{~F}$ & $156 / 94$ & ++ & 5.01 & Preeclampsia \\
\hline & T5 & 29 & 38 & 3200 & $\mathrm{~F}$ & $149 / 88$ & ++ & 4.91 & Preeclampsia \\
\hline
\end{tabular}


miRNA sequencing of exosomes and sequence analysis Human umbilical serum samples were obtained from 10 female volunteers $(5$ mild preeclampsia patients and 5 healthy volunteers). Illumina TruSeq RNA Sample Preparation Kit (catalog \# RS-122-2001) was used for library preparation. Illumina HiSeq $\mathrm{X}$ Ten platform was use to sequence libraries to obtain $150 \mathrm{bp}$ paired-end data (minimal depth of $30 \times$ base coverage). CASAVA tool (v1.8 Illumina, San Diego, CA, USA) was used to obtain "Fastq. File". Raw reads counts were normalized as Reads Per Kilobase of transcript per Million mapped reads (RPKM). miRNA sequences were analyzed using miRWalk (http://mirwalk.uni-hd.de) and miRBase 22.0 (http://www. mirbase.org) webservers FastQC tool was used to perform quality control of sequence reads (http://www.bioinforma tics.babraham.ac.uk/projects/fastqc/). pheatmap package in $\mathrm{R}$ was used to generate heatmaps. RNAhybrid (energy $<-25$ ) and miRanda (energy $<-20$, score $\geq 150$ ) tools were used predict potential miRNA targets. Significant protein expression, gene ontology (GO) and associated pathway were explored through bioinformatics analyses. Kyoto Encyclopedia of Genes and Genomes (KEGG) pathway and GO analyses were carried out using DAVID 6.8 tool (https://david.ncifcrf.gov) [14-17].

\section{Western blotting analysis}

Western blotting was carried out to quantify protein levels as previously reported [18]. Protein samples were subjected to $10 \%$ SDS-PAGE gel electrophoresis. After transfer to membranes, the proteins were probed with the following primary antibodies (CD63 (SBI EXOAB-CD63A-1, Mountain View, CA, USA, 1:1000), GAPDH (Beyotime AG0191, Shanghai, China, 1:1000) and secondary antibody (1: 1000). The proteins were visualized using the Minichemi Chemiluminescence Imaging Instrument (Sage, Beijing, China). This assay was carried out in triplicates.

\section{Data analysis}

The results are presented as means and standard deviations (SD) obtained from three experimental repeats, unless otherwise stated. Group comparisons were done using Student's t-test. $P<0.05$ indicated significant differences.

\section{Results}

\section{Extraction and analysis of serum-derived EXOs}

First, exosomes were isolated and their baseline characteristics were evaluated (Fig. 1). Presence of tetraspanin CD63 (Fig. 1A, left panel) implies that isolated vesicles were mainly exosomes. Characterization of these purified exosomes by NTA showed that their size ranged between $\sim 100.5 \pm 4.0$ (Fig. 1B). This finding matched with typical sizes of exosomes. Additionally, the morphology and size of the exosomes were also examined by TEM (scale bar, $200 \mathrm{~nm}$ ) (Fig. 1C).

\section{Analysis of differential expression of serum miRNAs in serum EXOs}

A total of 1733 known miRNAs were identified based on sequence similarity (Supplemental Table S1). The original sequencing data can be accessed on the NCBI SRA database: PRJNA706741. There were 157 mature miRNAs that were differentially expressed between samples from $P E$ and control groups $\left(\log _{2}|\mathrm{FC}|>1, P<0.05\right.$; Supplementary Table S2). Out of the 157 differentially expressed miRNAs, 96 were upregulated whereas 61 were downregulated and were presented in a volcano plot (Fig. 2).

\section{GO and pathway analyses for miRNA target genes}

Analysis of the 157 differentially expressed miRNAs showed that they can target 51,424 differentially expressed genes (Fig. S1, Supplemental Table S3). In addition, GO (Fig. 3) and KEGG pathway analyses (Fig. 4) were conducted to explore the roles of miRNA

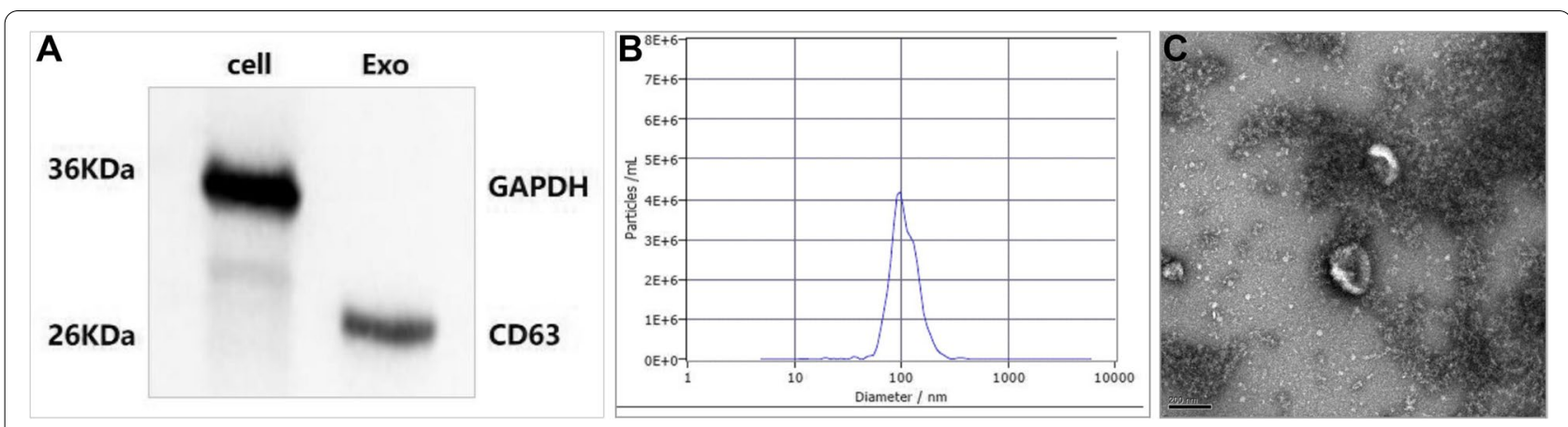

Fig. 1 Characteristics of human serum exosomes. A Western blot analysis of exosomes surface markers (CD63). B Representative nanoparticle tracking analysis (NTA) profile human serum exosomes C Serum-derived exosomes as observed under electron microscope (scale bar, $200 \mathrm{~nm}$ ) 

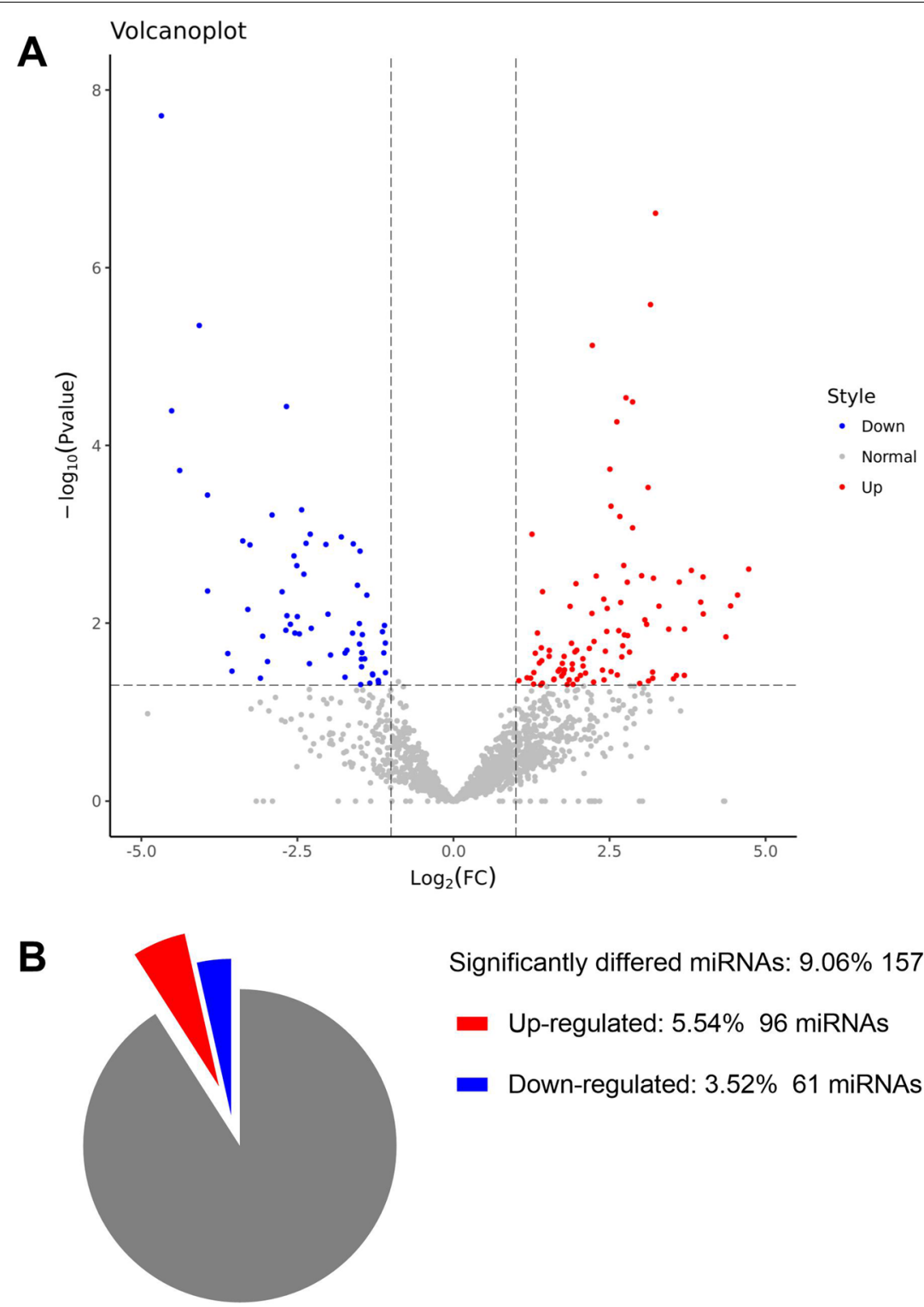

Significantly differed miRNAs: $9.06 \% 157$ miRNAs

Up-regulated: $5.54 \% 96$ miRNAs

Down-regulated: $3.52 \% 61$ miRNAs

Total=1733

Fig. 2 Cluster analysis of differentially expressed exosomal miRNAs isolated from serum of PE patients and normal controls. A Heat map showing hierarchical clustering (HCL) of normalized levels of the 157 differentially expressed microRNAs from PE $(n=5)$ and normal control $(n=5)$. B Volcano map showing distribution of differentially expressed microRNAs based on the $p$ values and fold-changes. $p<0.05$ and $\mid \log 2$ (fold-change) $\mid \geq 1$ were used to show differential expression

target genes. Results showed that the genes were linked to transcription, phosphorylation, development and angiogenesis. KEGG results revealed that the genes were linked to rap1 pathways, oxytocin pathways, MAPK and calcium signaling pathways.

\section{Discussion}

Preeclampsia is a complex pregnancy-related syndrome involving several factors and is linked to high risk of cardiovascular disease in pregnant mothers and their newborns [23]. In a previous study on preeclampsia patients, we carried out iTRAQ analysis on umbilical artery samples. The findings showed 53 differentially 


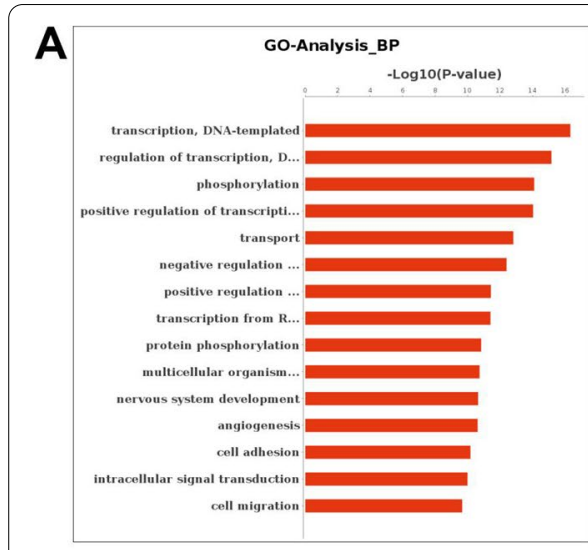

B

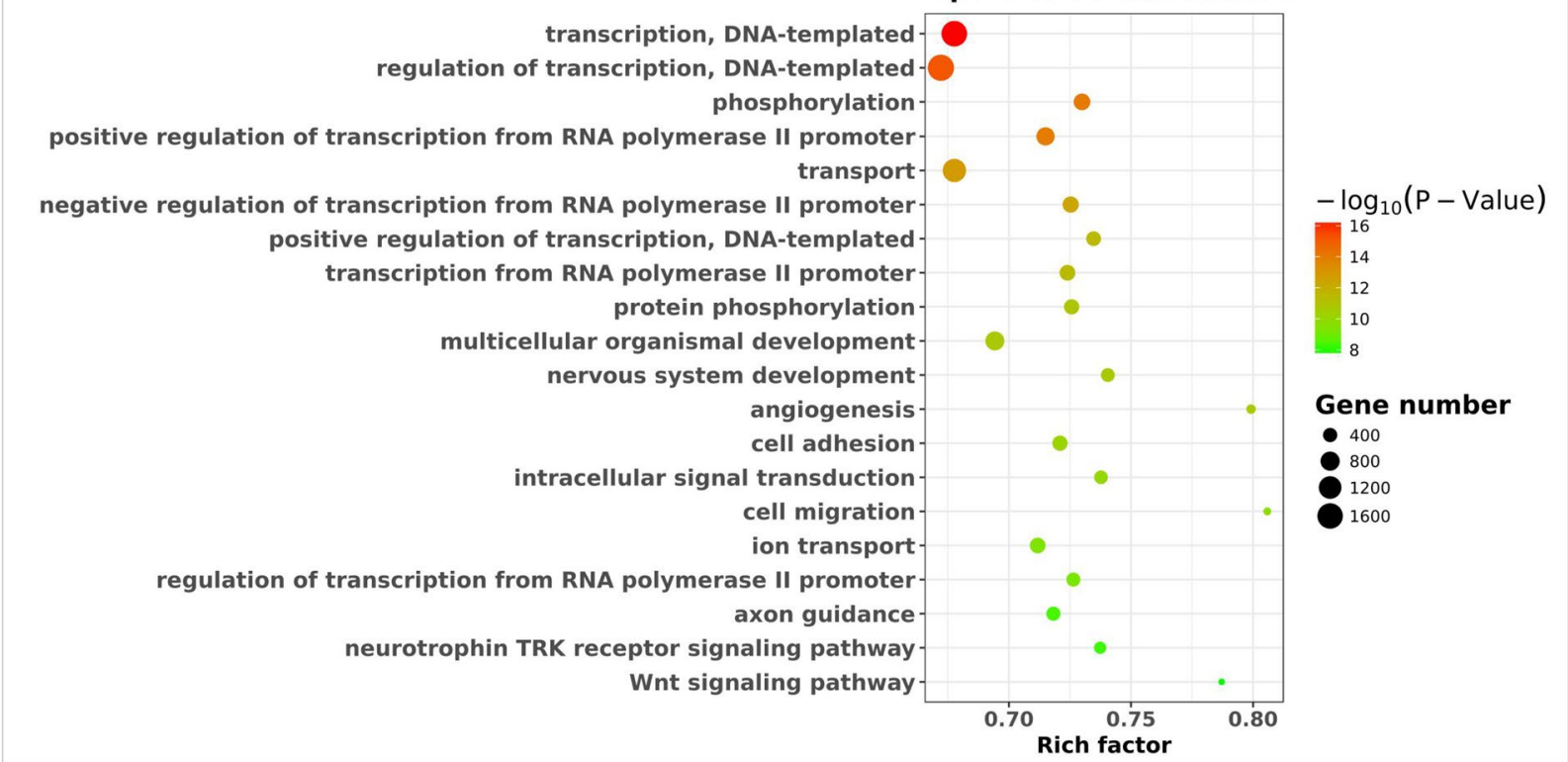

Fig. 3 Gene ontology (GO) enrichment analysis of predicted target genes. A GO analysis of differentially expressed genes for Biological Process (BP), Molecular Function (MF), and Cellular Component (CC) terms

expressed proteins in which participate in developmental processes of the cardiovascular system. Overall, 1733 known miRNAs were found in this study. Analysis of exosomal microRNAs sequence data showed 157 differentially expressed mature miRNAs in PE-derived serum exosomes compared with control group. Out of the 157 differentially expressed miRNAs, 96 were upregulated whereas 61 miRNAs were downregulated. Further analysis showed that the 157 differentially expressed miRNAs targeted 51,424 DEGs. Gene Ontology and KEGG pathway analyses showed significant enrichment of the target DEGs in several biological processes and pathways.

Umbilical cord blood is characterized by specific features compared with adult blood, therefore, studies explore it to understand the fetal state. In the present study, several significantly differentially expressed miRNAs related with cardiovascular function. miR-483-5p associates with obesity and cardiovascular disease [19], also ameliorates hypercholesterolemia by inhibiting PCSK9 production [20]. The miRNA miR-483-3p is an important modulator of endothelial integrity in type 2 diabetes (T2D) patients and is a potential therapeutic target for alleviating endothelial regeneration after injury T2Dpatients [21]. hsa-miR-1237-3p expression is correlated with certain fatty acids levels [22]. hsa-miR-365b-5p and hsa-miR-155-5p affectively modulates vascular functions and proliferation of HUVEC [19]. Moreover, hsa-miR-155-5p is a potential biomarker which for differentiating Hypertrophic cardiomyopathy (HCM) patients from healthy controls [23]. Changes in expression levels of miR-200b in acute hypoxia may exert a proangiogenic effect by downregulating Klf2 thus stabilizing HIF-1 signaling [24]. hsa-miR-200b-3p can be used as treatment targets for hypoxic-related and cardiovascular diseases 

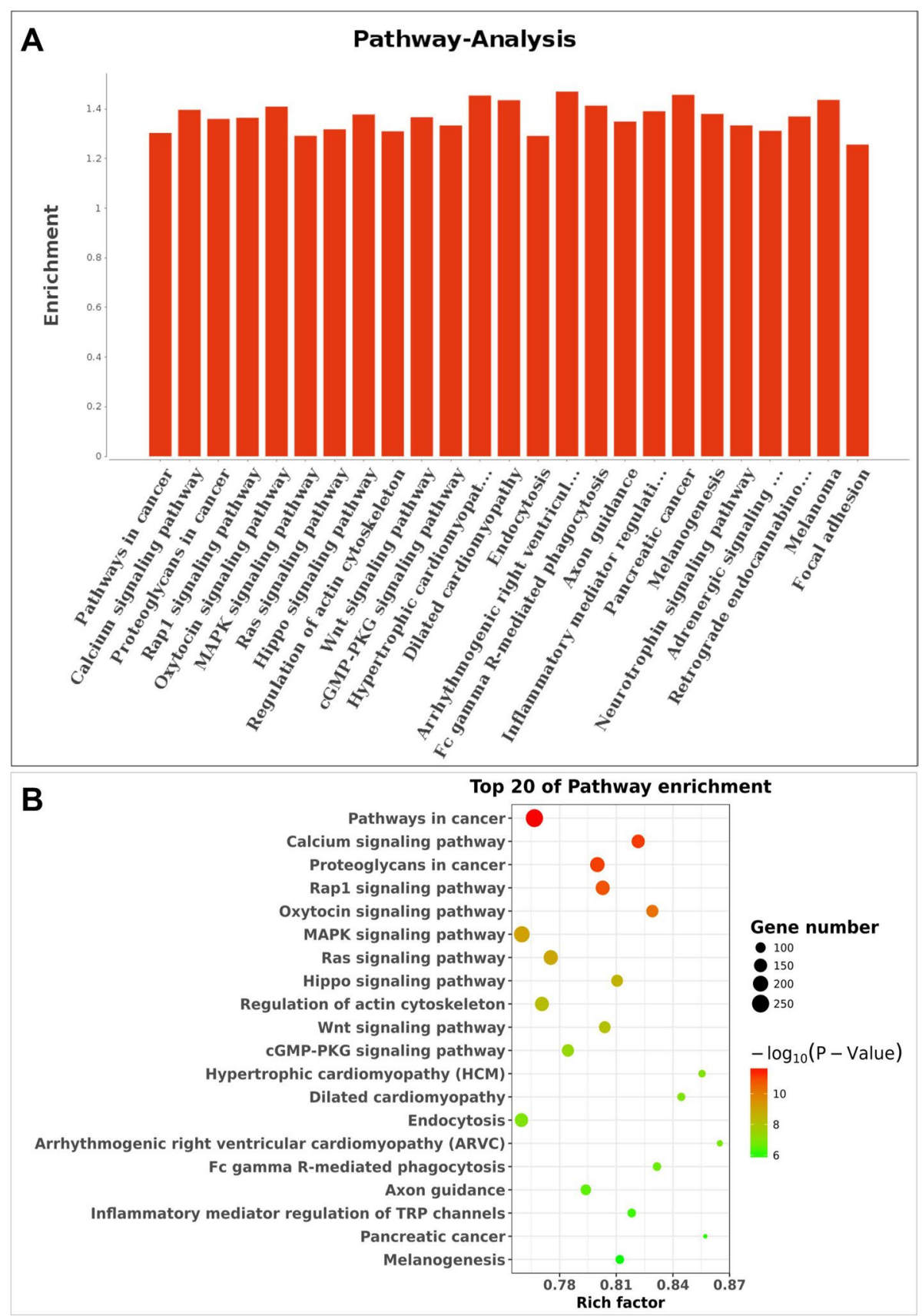

Fig. 4 KEGG pathway analysis for predicted target genes of differentially expressed miRNAs. A Coordinate axis X: $-\log _{10}(P$-value); coordinate axis Y: Pathway-Term entry name. B Coordinate axis $X:-\log _{10}(P$-Value); coordinate axis Y: Pathway-Term entry name

including different cancer types [25]. hsa-miR-342-3p is a potential biomarker in children with endothelial dysfunction [26]. Notably, hsa-miR-140-3p expression is downregulated in CAD (coronary artery disease) patients [27]. hsa-miR-3909 is correlated with rheumatic valvular heart disease [28]. However, the roles of different miRNA expressions in offspring microvascular dysfunction of preeclampsia remains have not been explored.
Several DEGs were identified that are targeted by differentially expressed miRNAs. GO and KEGG pathway analyses were used to explore potential biological functions and potential mechanisms of miRNA found in offspring microvascular dysfunction of preeclampsia. GO term analysis of these target genes showed a number of remarkably linked to GO terms associated with transcription, phosphorylation, development and 
angiogenesis. Multiple genetic disorders, mainly metabolic and cardiovascular diseases are associated with transcription of metabolism-associated genes [29]. Protein phosphorylation is a key signaling mechanism for regulating cellular pathways implicated in several physiological processes associated with cardiovascular and metabolic systems in the body [30]. Recently, epigenetics especially microRNAs have attracted growing attention in regulating embryonic vascular development [31]. Exosomal microRNAs are implicated in modulating endothelial cell function and angiogenesis [32].

Findings from KEGG pathway analysis uncovered that the target genes correlated with calcium rap1, oxytocin and MAPK signaling pathways. Calcium signaling plays a key role in cardiovascular excitability and function [33]. High expression levels of endogenous TRPM8 inhibits the function of Rap1 GTPase and plays an important role in modulation of activities of vascular endothelial cells by inhibiting migration [34]. Oxytocin also exerts cardiovascular and antiobesity effects through central and peripheral oxytocin receptor [35]. Crosstalk between p38 MAPK signaling and extracellular signal-related kinase (ERK) $1 / 2$ modulates various physiological functions, such as cardiac fibrosis and vascular smooth muscle cell migration [36].

\section{Conclusions}

Our findings, indicating the differential expression of umbilical serum Exo-miRNAs between normal and PE patients, reveal that these Exo-miRNAs may associate with microvascular dysfunction in offspring of mothers with preeclampsia.

\section{Abbreviations}

PE: Preeclampsia; miRNAs: microRNAs; GO: Gene ontology; KEGG: Kyoto Encyclopedia of Genes and Genomes.

\section{Supplementary Information}

The online version contains supplementary material available at https://doi. org/10.1186/s12884-022-04449-w.

Additional file 1: Figure S1. Venn diagram of target genes of differentially expressed miRNAs. RNAhybrid: Energy $<-25$. Miranda: score $>150$, Energy $<-20$.

Additional file 2: Figure S2. Original western blot gels for Fig. 1A.

Additional file 3: Table S1. A total of 1733 miRNAs were identified in serum exosomes from patients with PE and control groups.

Additional file 4: Table S2. 157 differentially expressed miRNAs in serum exosomes between the PE group and the control group.

Additional file 5: Table S3. Target genes of differentially expressed miRNAs.
Acknowledgements

Not applicable.

\section{Authors' contributions}

HT.P, M.F.: Writing - original draft, B.Y: Methodology, XL.S.: Formal analysis, PP.C: Visualization, XM.Sun: Validation, JL.D, GY.X: Methodology, HD.Z, T.Z: Project administration. All authors read and approved the final manuscript.

\section{Funding}

This work was supported by the National Natural Science Foundation of China (82071729), the China Postdoctoral Science Foundation (2020 M681336), the Science Technology Department of Zhejiang Province, China (LGF21H040004, LGF19H040004, LGD2OH040001) and the Health Commission of Zhejiang Province, China (2022RC277, 2022KY413, 2021 KY375, 2019RC296, 2019KY229, 2019KY717); the Science Technology Department of Shaoxing, China (2020A13034, 2020A13035, 2018C30050).

\section{Availability of data and materials}

The raw sequence reads are available under the SRA accession numbers PRJNA706741 [https://www.ncbi.nlm.nih.gov/sra/PRJNA706741].

\section{Declarations}

\section{Ethics approval and consent to participate}

The present study was approved by the Ethics Committee of Shaoxing Maternity and Child Health Care Hospital (Ethical Application Ref: 2018022). This study complied with the basic ethical principles contained in the Helsinki Declaration in its most recent version, from 2013. All participants signed a written informed consent form prior to their participation in this study.

\section{Consent for publication \\ Not applicable.}

\section{Competing interests}

The authors declare there are no competing interests.

\section{Author details}

${ }^{1}$ Shaoxing Maternity and Child Health Care Hospital, Shaoxing 312000, China. ${ }^{2}$ Obstetrics and Gynecology Hospital of Shaoxing University, Shaoxing, China. ${ }^{3}$ The International Peace Maternity and Child Health Hospital, School of Medicine, Shanghai Jiao Tong University, Shanghai 200030, China.

Received: 10 September 2021 Accepted: 3 February 2022

Published online: 14 February 2022

\section{References}

1. Yagel S, Cohen SM, Goldman-Wohl D. An integrated model of preeclampsia: a multifaceted syndrome of the maternal cardiovascular-placentalfetal array. Am J Obstet Gynecol. 2021. https://doi.org/10.1016/j.ajog. 2020.10.023.

2. Veiga ECA, et al. Previous preeclampsia and its association with the future development of cardiovascular diseases: a systematic review and metaanalysis. Clinics (Sao Paulo). 2021;76:e1999. https://doi.org/10.6061/clini CS/2021/e1999.

3. Dyess NF, Kinsella JP. Cardiovascular implications for offspring born to mothers with preeclampsia. J Pediatr. 2021;228:11-2. https://doi.org/10. 1016/j.jpeds.2020.08.015.

4. Deb A, Gupta S, Mazumder PB. Exosomes: a new horizon in modern medicine. Life Sci. 2021;264:118623. https://doi.org/10.1016/j.lfs.2020. 118623.

5. Liang Y, Duan L, Lu J, Xia J. Engineering exosomes for targeted drug delivery. Theranostics. 2021;11:3183-95. https://doi.org/10.7150/thno.52570.

6. Kalluri R, LeBleu VS. The biology, function, and biomedical applications of exosomes. Science. 2020;367. https://doi.org/10.1126/science.aau6977.

7. Napoli C, et al. Novel epigenetic-based therapies useful in cardiovascular medicine. World J Cardiol. 2016;8:211-9. https://doi.org/10.4330/wjc.v8.i2. 211. 
8. Maegdefessel L. The emerging role of microRNAs in cardiovascular disease. J Intern Med. 2014;276:633-44. https://doi.org/10.1111/joim.12298.

9. Levy E, Spahis S, Bigras JL, Delvin E, Borys JM. The epigenetic machinery in vascular dysfunction and hypertension. Curr Hypertens Rep. 2017;19:52. https://doi.org/10.1007/s11906-017-0745-y.

10. Zou K, Ding G, Huang H. Advances in research into gamete and embryofetal origins of adult diseases. Sci China Life Sci. 2019;62:360-8. https:// doi.org/10.1007/s11427-018-9427-4.

11. van Rijn BB, et al. Cardiovascular disease risk factors in women with a history of early-onset preeclampsia. Obstet Gynecol. 2013;121:1040-8. https://doi.org/10.1097/AOG.0b013e31828ea3b5.

12. Jayet PY, et al. Pulmonary and systemic vascular dysfunction in young offspring of mothers with preeclampsia. Circulation. 2010;122:488-94. https://doi.org/10.1161/CIRCULATIONAHA.110.941203.

13. Zhang F, et al. High throughput microRNAs sequencing profile of serum exosomes in women with and without polycystic ovarian syndrome. PeerJ. 2021;9:e10998. https://doi.org/10.7717/peerj.10998.

14. Huang da W, Sherman BT, Lempicki RA. Bioinformatics enrichment tools: paths toward the comprehensive functional analysis of large gene lists. Nucleic Acids Res. 2009;37:1-13. https://doi.org/10.1093/nar/gkn923.

15. Huang da W, Sherman BT, Lempicki RA. Systematic and integrative analysis of large gene lists using DAVID bioinformatics resources. Nat Protoc. 2009:4:44-57. https://doi.org/10.1038/nprot.2008.211.

16. Kanehisa M, Sato Y, Kawashima M, Furumichi M, Tanabe M. KEGG as a reference resource for gene and protein annotation. Nucleic Acids Res. 2016:44:D457-62. https://doi.org/10.1093/nar/gkv1070.

17. Kanehisa M, Furumichi M, Tanabe M, Sato Y, Morishima K. KEGG: new perspectives on genomes, pathways, diseases and drugs. Nucleic Acids Res. 2017;45:D353-d361. https://doi.org/10.1093/nar/gkw1092.

18. Pan $\mathrm{HT}$, et al. Proteomics and bioinformatics analysis of altered protein expression in the placental villous tissue from early recurrent miscarriage patients. Placenta. 2018;61:1-10. https://doi.org/10.1016/j.placenta.2017. 11.001.

19. Kasiviswanathan D, et al. Interactome of miRNAs and transcriptome of human umbilical cord endothelial cells exposed to short-term simulated microgravity. NPJ Microgravity. 2020;6:18. https://doi.org/10.1038/ s41526-020-00108-6.

20. Dong J, et al. microRNA-483 ameliorates hypercholesterolemia by inhibiting PCSK9 production. JCI Insight. 2020;5. https://doi.org/10.1172/jci.insig ht.143812.

21. Kuschnerus K, et al. Increased expression of miR-483-3p impairs the vascular response to injury in type 2 diabetes. Diabetes. 2019;68:349-60. https://doi.org/10.2337/db18-0084.

22. Raitoharju E, et al. Blood microRNA profile associates with the levels of serum lipids and metabolites associated with glucose metabolism and insulin resistance and pinpoints pathways underlying metabolic syndrome: the cardiovascular risk in young Finns study. Mol Cell Endocrinol. 2014;391:41-9. https://doi.org/10.1016/j.mce.2014.04.013.

23. Shi $\mathrm{H}$, et al. Systematic identification and analysis of dysregulated miRNA and transcription factor feed-forward loops in hypertrophic cardiomyopathy. J Cell Mol Med. 2019;23:306-16. https://doi.org/10.1111/jcmm. 13928.

24. Bartoszewski R, et al. miR-200b downregulates Kruppel like factor 2 (KLF2) during acute hypoxia in human endothelial cells. Eur J Cell Biol. 2017:96:758-66. https://doi.org/10.1016/j.ejcb.2017.10.001.

25. Janaszak-Jasiecka A, et al. eNOS expression and NO release during hypoxia is inhibited by miR-200b in human endothelial cells. Angiogenesis. 2018;21:711-24. https://doi.org/10.1007/s10456-018-9620-y.

26. Khalyfa A, Kheirandish-Gozal L, Bhattacharjee R, Khalyfa AA, Gozal D. Circulating microRNAs as potential biomarkers of endothelial dysfunction in obese children. Chest. 2016;149:786-800. https://doi.org/10.1378/chest. 15-0799.

27. Taurino $C$, et al. Gene expression profiling in whole blood of patients with coronary artery disease. Clin Sci (Lond). 2010;1 19:335-43. https://doi.org/ $10.1042 / C S 20100043$

28. Lu Q, et al. Comprehensive microRNA profiling reveals potential augmentation of the IL1 pathway in rheumatic heart valve disease. BMC Cardiovasc Disord. 2018;18:53. https://doi.org/10.1186/s12872-018-0788-2.

29. Duddu S, Chakrabarti R, Ghosh A, Shukla PC. Hematopoietic stem cell transcription factors in cardiovascular pathology. Front Genet. 2020;11:588602. https://doi.org/10.3389/fgene.2020.588602.
30. Senis YA, Barr AJ. Targeting receptor-type protein tyrosine phosphatases with biotherapeutics: is outside-in better than inside-out? Molecules. 2018;23. https://doi.org/10.3390/molecules23030569.

31. Guo Z, Mo Z. Regulation of endothelial cell differentiation in embryonic vascular development and its therapeutic potential in cardiovascular diseases. Life Sci. 2021:119406. https://doi.org/10.1016/j.lfs.2021.119406.

32. Kir D, Schnettler E, Modi S, Ramakrishnan S. Regulation of angiogenesis by microRNAs in cardiovascular diseases. Angiogenesis. 2018;21:699-710. https://doi.org/10.1007/s10456-018-9632-7.

33. Liao Q, et al. A novel ShK-like toxic peptide from the Transcriptome of the cnidarian Palythoa caribaeorum displays Neuroprotection and Cardioprotection in Zebrafish. Toxins (Basel). 2018;10. https://doi.org/10.3390/toxin s10060238.

34. Genova T, et al. TRPM8 inhibits endothelial cell migration via a nonchannel function by trapping the small GTPase Rap1. J Cell Biol. 2017;216:2107-30. https://doi.org/10.1083/jcb.201506024.

35. Bytautiene Prewit E, Kechichian T, Okunade D, Yin H, Stuebe AM. Effect of Normal pregnancy followed by lactation on long-term maternal health in a mouse model. Reprod Sci. 2018;25:1186-96. https://doi.org/10.1177/ 1933719117734316.

36. Koga Y, et al. Roles of cyclic AMP response element binding activation in the ERK $1 / 2$ and p38 MAPK Signalling pathway in central nervous system, cardiovascular system, osteoclast differentiation and Mucin and cytokine production. Int J Mol Sci. 2019;20. https://doi.org/10.3390/ijms20061346.

\section{Publisher's Note}

Springer Nature remains neutral with regard to jurisdictional claims in published maps and institutional affiliations.

Ready to submit your research? Choose BMC and benefit from

- fast, convenient online submission

- thorough peer review by experienced researchers in your field

- rapid publication on acceptance

- support for research data, including large and complex data types

- gold Open Access which fosters wider collaboration and increased citations

- maximum visibility for your research: over $100 \mathrm{M}$ website views per year

At BMC, research is always in progress.

Learn more biomedcentral.com/submissions 